\title{
THE UNACKNOWLEDGED IMPACT OF URINARY SCHISTOSOMIASIS IN CHILDREN: 5 CASES FROM KUMASI, GHANA
}

\author{
S. ANTWI ${ }^{1}$, K. E. K. ABOAH ${ }^{2}$ and C. K. G. SARPONG ${ }^{3}$ \\ ${ }^{1}$ Department of Child Health and ${ }^{2}$ Department Of Surgery, School Of Medical Sciences, Kwame Nkrumah \\ University of Science and Technology/Komfo Anokye Teaching Hospital, Kumasi, Ghana
}

DOI: http://dx.doi.org/10.4314/gmj.v48i4.11

Corresponding Author: Dr Sampson Antwi

Email address: kantwisampson@gmail.com

Conflict of interest: None declared

\begin{abstract}
SUMMARY
Urinary schistosomiasis is a parasitic disease caused by Shistosoma haematobium. It is prevalent in several parts of Africa particularly in areas where there are large water bodies. In most affected communities, the condition is often accepted as normal since to them, all growing children pass blood in their urine and "grow out of it". Mass treatment of school children has been a regular exercise often undertaken by stake holders to decrease the disease burden and reduce transmission in selected communities.
\end{abstract}

Urinary schistosomiasis can have devastating impact on the urinary tract which is often unacknowledged and unevaluated. Such omission could have implication for progressive renal damage which, if not detected and treated, could lead to end stage renal failure and death.

We present five (5) cases of urinary schistosomiasis with severe obstructive uropathy seen at the paediatric nephrology/urology units of Komfo Anokye Teaching Hospital, Ghana. All five cases had some degree of anaemia and hypertension. Two of the five cases presented with end stage renal failure and died subsequently whilst two underwent successful surgery. One made a spontaneous recovery from the urinary obstruction though still has significant renal impairment.

This potential devastating effect of urinary schistosomiasis on the kidneys calls for thorough evaluation and assessment of each confirmed case to include blood pressure measurement, full blood count, and ultrasonography of the urinary system. Mass screening programmes should be combined with portable ultrasonography of the kidneys, ureters and bladder.

Keywords: Urinary schistosomiasis, Obstructive uropathy, End Stage Renal-Failure, Hydroureteronephrosis, Nephrostomy.

\section{INTRODUCTION}

Urinary schistosomiasis is a common parasitic disease affecting hundreds of millions of people in many countries in the tropics. ${ }^{1-3}$ It is caused by the blood fluke Schistosoma haematobium. The disease is prevalent in areas where there are large water bodies for irrigation and particularly where dams have been constructed. ${ }^{1,4}$

In Ghana, the disease is endemic and widely distributed in many areas around the major rivers. ${ }^{5,6}$ Prevalence rate of $12.4 \%$ has been reported in some endemic regions in Ghana. ${ }^{6}$ Because of the lifestyle and behaviour of children in swimming in large water bodies, they are the most at risk of the disease. ${ }^{2,7}$ The disease manifests principally as passage of haematuria, overt (terminal or total) or covert. In endemic regions, there is a high prevalence of haematuria among school age children to the extent that children who do not pass blood in their urine may be regarded as "abnormal" by their peers. ${ }^{2}$ The infection is confirmed by the identification of the ova (with a lateral spine) on urine microscopy.

The widespread endemicity of this disease and its familiarity in such regions of the world often tend to downplay the devastating impact that this disease can cause. Quite often, individual clinical cases are only treated with praziquantel (the drug of choice) without recourse to further evaluation and investigations. In Ghana, several mass treatment programmes with praziquantel have been directed at school children in endemic areas without further assessment for complications. But urinary schistosomiasis can have devastating impact on the urinary tract and indeed the whole kidney which, if not detected early and prompt interventions instituted, could be fatal. ${ }^{2,8}$ This, notwithstanding, in most endemic countries, the extent of renal complications of schistosomiasis in children has not been fully established. ${ }^{2}$ It is believed to be higher than generally appreciated. ${ }^{9}$ 
The most prominent and important pathology in schistosomiasis results from the host response to schistosomal eggs retained in tissues ${ }^{2,8,9}$ Following infection, the adult worms (male: female pairings) inhabit the venules of the urinary tract from where the females release large quantities of eggs which may remain embedded in the tissues, embolise to other organs, or pass into the urine. ${ }^{8}$ Eggs trapped in tissues stimulate a strong granulomatous reaction which heals by fibrosis. $^{2,8,9}$

On the urinary system, schistosomal nephropathy typically presents as granulomatous inflammation of the distal third of both ureters, the urinary bladder and the urethra and may be followed by calcification resulting in hydroureters, hydronephrosis, and bladder neck obstruction. ${ }^{2,8,9}$ In the bladder, collections of fine mucosal tubercles called sandy patches may develop as well as granulomas of various sizes (sessile or pedunculated). ${ }^{2}$ Persistent bladder schistosomiasis may be linked to squamous cell carcinoma of the bladder in adulthood. ${ }^{2,3}$ Besides its effect on the urinary tract, schistosomal nephropathy may also present as immune-complex mediated glomerular disease typically as nephrotic syndrome. $2,8-10$

The chronic loss of blood that ensues from the persistent symptom of haematuria (overt or covert) may be a cause of iron deficiency anaemia and poor growth in children which may assume public health dimensions in heavily infected communities. ${ }^{2,11}$ The effect of urinary schistosomiasis is influenced by both the intensity of infection (judged by the eggs count) and the duration of infection ${ }^{2,8,9}$

In this article, we present five of eight cases of urinary schistosomiasis that were seen in the paediatric nephrology/urology units of Komfo Anokye Teaching Hospital (KATH) over a 2-year period. All five cases presented with obstructive uropathy, two of whom died from their disease; two underwent successful surgery; and one made a spontaneous "recovery". The other three cases (not presented in this article) presented with acute glomerulonephritis-like syndrome and made complete recovery.

\section{Case 1}

A 7-year old girl, EO, who had been admitted to the paediatric emergency unit of KATH with congestive heart failure (bibasal crepitations, cardiomegaly, tender hepatomegaly and peripheral oedema) and hypertension (BP 180/120mmHg) was subsequently found to have ova of Schistosoma haematobium in her urine. She had lived in Akosombo for greater part of her life.
Apart from the hepatomegaly, no other organ was palpable on abdominal examination. The urinary bladder was not palpable either.

Bedside urine dipstick showed protein $4+$, blood $2+$. Laboratory results were as follows: $\mathrm{Hb} 9.8 \mathrm{mg} / \mathrm{dl}, \mathrm{MCV}$ 65 fl, WBC 6.7 x 109/1, Platelet 217 x 109/1, Serum chemistry: sodium $(\mathrm{Na}+) 128 \mathrm{mmol} / 1$, potassium $(\mathrm{K}+)$ $4.2 \mathrm{mmol} / 1$, urea $15 \mathrm{mmol} / 1$, creatinine $430 \mu \mathrm{mol} / 1$, albumin $36 \mathrm{~g} / 1$, total protein $73 \mathrm{~g} / 1$.

Urine culture isolated E. coli. Chest X-ray (CXR) showed cardiomegaly with pulmonary oedema and bilateral pleural effusion.

Ultrasonography scan (USS): showed bilaterally dilated calyces with bilateral hydroureters. The bladder wall was diffusely thickened. Diagnoses of acute kidney injury (AKI) secondary to schistosomal related obstructive uropathy with urinary tract infection, and congestive cardiac failure (CCF) secondary to severe hypertension were made.

Patient was appropriately treated with frusemide, amlodipine, praziquantel and antibiotics. Indwelling urethral catheter was passed to monitor the urine output and to relieve possible bladder neck obstruction from the schistosomiasis. Urine output in excess of $2 \mathrm{mls} / \mathrm{kg} / \mathrm{hr}$ was recorded in the subsequent 24 hours. Following the above treatment, the kidney function test normalised with serum creatinine falling to $58 \mu \mathrm{mol} / 1$. On discharge, she was already micturiting freely without urethral catheter in-situ.

Patient was discharged to be followed up on outpatient basis and to do combined micturiting cystourethrogram (MCUG) and intravenous urogram (IVU). The radiological evaluations of the urinary tract could not be done for technical and logistic reasons and patient presented 3 months later with generalised bodily swelling and decreased urine output. She was re-admitted.

On re-admission, BP and other cardiovascular findings were normal. Abdominal examination revealed no organomegaly and no palpable bladder. Urine output was, however, low $(0.1 \mathrm{ml} / \mathrm{kg} / \mathrm{hr})$ over the first 12 hours of re-admission. Serum chemistry results were $\mathrm{Na}+$ $121 \mathrm{mmol} / 1, \mathrm{~K}+4.2 \mathrm{mmol} / 1$, urea $63 \mathrm{mmmol} / 1$, creatinine $732 \mu \mathrm{mol} / 1$, calcium $1.3 \mathrm{mmol} / 1$, phosphate $3.6 \mathrm{mmol} / 1$

Urethral catheter was re-introduced and high dose frusemide infusion $(0.5 \mathrm{mg} / \mathrm{kg} / \mathrm{hr})$ instituted. Her urine output improved only marginally to $0.4 \mathrm{ml} / \mathrm{kg} / \mathrm{hr}$ in the subsequent days whilst serum creatinine fluctuated between 1500 and $500 \mu \mathrm{mol} / 1$. 
IVU to assess the patency of the distal ureters could not be done on account of the unresolved abnormal renal function. Cystoscopic examination could also not be done.

Six weeks into admission, surgical exploration of the urinary tract was embarked upon after an attempted insertion of nephrostomy tube led to kidney haemorrhage.

Intra-operative findings were as follows: The distal ends of both ureters were much thickened with calcification. Both ureteric orifices were severely stenosed and could not be visualised. Bladder was uniformly thickened with sandy patches. Bilateral ureteroneocystostomy was done with placement of stent.

Following these procedures the urine output did not improve and patient died on 3rd post operative day from severe renal failure and recalcitrant metabolic acidosis.

\section{Case 2}

Twelve-year-old R. O. from Nkoranza was referred to KATH as a possible seizure disorder. He had presented to a local hospital with fever, severe headache and several episodes of generalised convulsions.

The child was fully conscious but had a BP of 140/94mmHg (both systolic and diastolic values $>99$ th centile +5 for his age). Bedside urine dipstick showed protein and blood of $2+$ each.

Baseline laboratory test results were as follows: Serum chemistry: $\mathrm{Na}+136, \mathrm{~K}+5.6$, urea 16.3 , creatinine $389 \mu \mathrm{mol} / 1$, Hb $10.5 \mathrm{~g} / \mathrm{dl}, \mathrm{MCV} 72 \mathrm{fl}, \mathrm{WBC} 9.9 \times 10^{9} / 1$, platelet $444 \times 10^{9} / 1$

Urine microscopy showed pus cells 5-6/HPF, RBCs > $10 / \mathrm{HPF}$, and $S$. haematobiuon ova were present. Urine culture result was negative. USS showed a lobulated, hypoechoeic urinary bladder trigone mass with resultant bilateral hydroureteronephrosis.

Diagnoses of hypertensive encephalopathy and acute kidney injury secondary to schistosoma related obstructive uropathy were made. He was treated with praziquantel and amlodipine.

A scheduled nephrostomy tube insertion and cystoscopic excision of the trigone mass could not be done as patient defaulted follow up for several months. When he re-surfaced 6 months later, there had been spontaneous improvement in his renal function with the serum creatinine falling to $150 \mu \mathrm{mol} / \mathrm{l}$. The trigone mass has reduced in size and the left hydrouretero- nephrosis had resolved completely. BP had been well controlled on amlodipine. He was then scheduled for 6monthly review to assess progress.

In the subsequent six months, all the hydroureteronephrosis had resolved spontaneously. The left kidney had shrunken somewhat, measuring $6.3 \times 2.7 \mathrm{~cm}$ but with good cortico-medullary differentiation. Right kidney measured $8.3 \times 3.3 \mathrm{~cm}$. A residual mass in the trigone area had remained. The kidney function had stabilised with serum creatinine at $130 \mu \mathrm{mol} / \mathrm{l}$ and urea of $4.1 \mathrm{mmol} / 1$.

He was put on lisinopril to control his proteinuria and slated for another 6 months review as a case of Stage 3 Chronic Kidney Disease (eGFR $52.7 \mathrm{ml} / \mathrm{min} / 1.73 \mathrm{~m}^{2}$ ).

\section{Case 3}

Twelve-year-old O. A. from Yeji, a schistosomiasis endemic area, presented with recurrent abdominal pain. He has had on and off terminal haematuria since age 4 years.

Essential findings on physical examination were moderate pallor, BP $127 / 90 \mathrm{mmHg}$ (systolic $>95$ th centile, diastolic $>99$ th centile), and fullness at the left flank with some tenderness. Bedside urine dipstick showed protein of $1+$ and blood of $3+$.

Initial laboratory findings were as follows: Urine microscopy; pus cells too numerous to count, RBCs $>$ $100 / \mathrm{HPF}$, S. haematobium ova $2+$; urine culture isolated E. coli sensitive to ciprofloxacin and ceftazidime; $\mathrm{Hb} 8.7 \mathrm{~g} / \mathrm{dl}$, MCV $73 \mathrm{fl}$; blood urea $1.9 \mathrm{mmol} / 1$, creatinine $20 \mu \mathrm{mol} / \mathrm{l}$, Na $139 \mathrm{mmol} / \mathrm{l}, \mathrm{K} 4.0 \mathrm{mmol} / \mathrm{l}$. USS showed bilateral hydroureteronephrosis with diffusely thickened urinary bladder wall more prominent posteriorly and fundally.

MCUG showed severe narrowing of the distal half of the posterior urethra with attendant dilatation of the proximal prostatic urethra. No vesicoureteric reflux was observed. IVU showed bilateral hydroureteronephrosis with moderate bladder filling implying distal ureteral involvement.

The urinary tract infection (UTI), hypertension and schistosomiasis were appropriately treated with antibiotics, amlodipine and praziquantel respectively. Patient was also given Iron for 3 months. Bilateral nephrostomy tubes were insertion and urethral catheter passed.

He subsequently underwent surgery for bilateral ureteric re-implantation. Intra-operative findings showed bilaterally thickened distal ureters with stenosis and a thickened urinary bladder. 
Follow up urine culture was sterile and BP was controlled. Repeat USS showed moderate dilatation of right renal pelvis and calyces and no evidence of dilated renal calyces or pelvis on the left.

Patient is doing well and is on long term follow up. He has indwelling urethral catheter in place and has been scheduled for urethrocystoscopy to fully evaluate the lower urinary tract and to rule out missed posterior urethral valve.

\section{Case 4}

Eleven-year-old I.T. was referred from a district hospital with provisional diagnosis of chronic renal failure. Patient hails from Suhum, a community endemic for schistosomiasis. His presenting complaints included bodily swelling, cough, and chest pain of a week's duration and terminal haematuria for a year.

Essential findings on physical examination were generalised oedema, pallor, dyspnoea, respiratory rate of $36 / \mathrm{min}$ and bilateral basal crepitations. The pulse rate was $112 / \mathrm{min}$, BP 140/100mmHg (both systolic \& diastolic $>99$ th centile for age \& sex), heart sounds S1S2 were present and normal, there was no murmur. There was non-tender hepatomegaly of $3 \mathrm{~cm}$. The bladder was not palpable. Bedside urine dipstick showed protein $3+$ and blood $4+$.

Laboratory test results were as follows: $\mathrm{Hb} 8 \mathrm{~g} / \mathrm{dl}$, MCV 75 fl., WBC $6.5 \times 10^{9} / 1$ Platelet count of 220 x $10 \%$; Urine microscopy showed pus cells $>20 / \mathrm{HPF}$, RBCs > 100/HPF, granular cast 2+; S. haematobium ova ++ ; urine culture was negative; blood urea $37 \mathrm{mmol} / 1$, creatinine $786 \mu \mathrm{mol} / 1, \mathrm{Na}+121 \mathrm{mmol} / 1, \mathrm{~K}+$ $5.7 \mathrm{mmol} / \mathrm{l}, \mathrm{Ca}^{2}+1.8 \mathrm{mmol} / 1, \mathrm{Mg}^{2}+0.9 \mathrm{mmol} / 1$, PO4- $1.4 \mathrm{mmol} / 1$, albumin $32 \mathrm{~g} / 1$, total protein $70 \mathrm{~g} / 1$ and cholesterol $5.7 \mathrm{mmol} / 1$.

CXR showed cardiomegaly with pulmonary oedema and USS showed severe bladder wall thickening with right hydroureteronephrosis. Left kidney was shrunken with increased echogenicity and loss of corticomedullary differentiation

Diagnoses of congestive cardiac failure $2^{\circ}$ hypertension, and chronic renal failure $2^{\circ}$ obstructive uropathy from chronic urinary schistosomiasis were made.

Heart failure was appropriately treated with high dose frusemide, and hypertension treated with amlodipine. Urethral catheter was passed. Urine output over 24 hours into admission was $0.48 \mathrm{mls} / \mathrm{kg} / \mathrm{hr}$. Intended nephrostomy tube insertion could not immediately be done due to logistic constraint. Child passed away 72 hours into admission.

\section{Case 5}

Twelve-year-old H. M. was referred to KATH from a district hospital with left flank pain and passage of scanty urine for 4 days. She had been given IV fluids and IV frusemide at the district hospital on account of the oliguria yet urethral catheterisation yielded no urine. The flank pain had worsened subsequently with child wailing loudly that prompted urgent referral to KATH. Patient had lived in Krachie near Akosombo for first 8 years of life where she experienced recurrent terminal haematuria for 4 yrs.

Essential findings on physical examination were: BP $170 / 120 \mathrm{mmHg}$; the rest of cardiovascular examination was normal. Chest was clinically clear. There was tenderness in the left lumbar region but the kidneys and bladder were not palpable. There was no peripheral oedema.

Accompanying lab results showed $\mathrm{Hb} 9.7 \mathrm{~g} / \mathrm{dl}$., MCV $75 \mathrm{fl}$, serum creatinine $1,947 \mu \mathrm{mol} / 1, \mathrm{Na}+128 \mathrm{mmol} / 1$, $\mathrm{K}+7.3 \mathrm{mmol} / \mathrm{l}$; urinalysis of protein $2+$, blood $2+$, pus cell $6-8 / \mathrm{HPF}, \mathrm{RBC}>20 / \mathrm{HPF}, S$. haematobium ova $2+$; urine culture was negative.

Salbutamol nebulisation 5mg hourly and sodium polysterene (kayexalate) per rectum were instituted to control the hyperkalaemia. IV hydralazine was started with oral amlodipine. Urethral catheter passed yielded no urine.

Laboratory results were: blood urea of $52.9 \mathrm{mmol} / 1$, creatinine $2,282 \mu \mathrm{mol} / 1, \mathrm{Na}+127 \mathrm{mmol} / 1$ and $\mathrm{K}+$ $7.2 \mathrm{mmol} / 1$.

USS showed severe bilateral hydroureteronephrosis with left peri-nephric collection. Bladder was empty with drainage catheter in-situ. Diagnoses of acute kidney injury $2^{\circ}$ schistosomal related obstructive uropathy, and worsening hydronephrosis from IV fluids were made.

Nephrostomy tubes were inserted into both kidneys. Urine output per nephrostomy tubes for the first 24 hours yielded $9.3 \mathrm{mls} / \mathrm{kg} / \mathrm{hr}$. Fluid replacement in excess of $4 \mathrm{ml} / \mathrm{kg} / \mathrm{hr}$ of urine output was instituted. Patient also received full treatment with praziquantel.

BP was controlled, and flank pain decreased substantially. Good recovery of kidney function was recorded three weeks after placement of the nephrostomy tubes with the following laboratory results:

Three weeks into placement of nephrostomy tubes, new laboratory values of blood urea $5.8 \mathrm{mmol} / 1$, creatinine $93 \mu \mathrm{mol} / \mathrm{l}, \mathrm{Na}+135 \mathrm{mmol} / \mathrm{l}$ and $4.5 \mathrm{~K}+\mathrm{mmol} / \mathrm{l}$ 
were obtained. Anterograde pyelogram confirmed severe bilateral ureteral obstruction.

Patient subsequently underwent bilateral ureteral reimplantation with placement of double J-stent. Intraoperative findings showed fibrotic bilateral distal ureters none of which was "passable" at the distal $2-3 \mathrm{~cm}$ segment from the vesico-ureteric junction. Bladder wall was markedly thickened.

Patient has done well postoperatively and is still being followed up. An interval IVU is planned to assess the success and patency of the distal ureters.

\section{DISCUSSION}

The five cases presented in this article confirm the often unacknowledged, yet devastating impact of urinary schistosomiasis on the kidneys and urinary systems beyond the well known symptom of passage of terminal haematuria; and also on the haematological system (anaemia).

These cases show the full spectrum of pathologies exerted on the urinary system by schistosomiasis. All five cases presented with obstructive uropathy with various degrees of renal dysfunction.

Case 1 showed fibrosis with calcification of the distal ends of both ureters and uniform bladder thickening with sandy patches; Case 2 presented with granulomatous mass in the trigone of the urinary bladder (likely a bilhazioma) that led to obstructive uropathy; Case 3 had bilaterally stenosed distal ureters with bilateral hydroureteronephrosis and bladder outlet obstruction; Case 4 had a shrunken left kidney with hydroureteronephrosis on the right and severe bladder wall thickening secondary to bladder outlet obstruction; whilst case 5 showed bilaterally stenosed distal ureters with thickened bladder.

Cases 1 and 4 presented with end-stage renal failure (ESRF) at ages 7 and 11 years respectively and died consequently, emphasising the point that urinary schistosomiasis may not be benign after all. ${ }^{2,3,8,9}$ For Case 1, inability to do combined MCUG and IVU on initial discharge as well as the overly long delay in getting surgical intervention might have contributed to her untimely demise. For Case 4, the longstanding obstructive uropathy had caused chronic renal failure, secondary hypertension and consequent heart failure. The high blood pressure, which had not been detected earlier, could have accelerated the progression of the kidney injury.
The existence of various degrees of urinary tract obstruction among these five cases of urinary schistosomiasis stress the need for some form of radiological evaluation such as ultrasonography beyond the diagnosis and treatment with praziquentel. ${ }^{2,9,11}$

Case 2 showed spontaneous resolution of the bladder granuloma and confirms previous literature reports ${ }^{2}$ though significant renal dysfunction still exists.

Cases 3 and 5 that had prompt relief of their obstructive uropathies by way of nephrostomy and subsequent ureteric re-implantation made dramatic improvement in their renal function. The two have thus been saved from potential fatality emphasising the need for prompt diagnosis and treatment of schistosomal renal complications.

All the five cases presented in this article had some form of iron deficiency anaemia (haemoglobin < $11.5 \mathrm{~g} / \mathrm{dl}$ with low MCV) that was likely contributed to by the chronic loss of blood in the urine though other confounding factors were not ruled out. Urinary schistosomiasis has long been reported to be associated with anaemia among school going children. ${ }^{11}$

None of the five cases had the acute urinary symptoms of overt haematuria, dysuria and urinary frequency at the time of admission. However, all five had ova of $S$. haematobium in the urine. Thus the resolution of acute symptoms of haematuria, dysuria and urinary frequency may not constitute a cure. Once previously infected with schistosomiasis, further evaluation may be warranted to rule out the long-term complications in the urinary tract. ${ }^{11}$

All five cases had either lived in recognised schistosomiasis endemic areas in Ghana or had had contact with large water bodies. ${ }^{4}$ The other three cases of urinary schistosomiasis seen at the nephrology unit (not discussed in this article) presented with glomerulopathy in the form of nephritic syndrome confirming previous reports. ${ }^{2,8-10}$

The obstructive uropathy that may ensue from the fibrotic changes of the urinary tract often leads to hypertension. ${ }^{12}$ This was evident in all the five cases with Cases 1 and 2 presenting with hypertensive encephalopathy and Case 4 with congestive heart failure. Thus there is the need to check the blood pressure of any child with obstructive uropathy to rule out hypertension.

Obstructive uropathy may also predispose to $\mathrm{UTI}^{2}$ In the presence of a closed urinary system like obstructive 
uropathy UTI could have a devastating effect on the kidneys and may lead to renal failure.

Such might have been the situation with Case 1 on first admission when the renal failure resolved with treatment of the UTI. UTI itself could lead to scarring of the kidneys and further predispose to hypertension. Both hypertension and UTI could worsen kidney function if not promptly detected and treated.

\section{CONCLUSION}

Urinary schistosomiasis may not be benign after all as the approach to management in clinical practice seems to suggest. Rather, urinary schistosomiasis could have devastating impact on the urinary system as demonstrated in these five cases. In the light of these potentially devastating effects of urinary schistosomiasis, management of urinary schistosomiasis should not be limited to treatment with praziquantel only. Rather, all cases of confirmed urinary schistosomiasis should have some further evaluation including full blood count, urine culture \& sensitivity, blood urea, creatinine; and radiological evaluation of the kidneys, ureters and urinary bladder. In the era of technological advancement, mass screening exercises for urinary schistosomiasis could be coupled with portable ultrasonography of the urinary system so that persons with established complications of obstructive uropathy could be identified and referred. Affected children should also have their blood pressures measured to rule out hypertension.

Such evaluation could help in the early identification and treatment of renal complications of schistosomiasis to forestall ongoing kidney injury. This becomes imperative particularly in Africa where the disease is endemic and where renal replacement therapy for the management of ESRF may either not be available or may be beyond the purchasing power of the average African.

\section{ACKNOWLEDGEMENT}

The staffs of the Paediatric Nephrology Unit and Urology Unit of KATH deserve commendation for the various ways they provided support in patients' care. We are also grateful to the staff of the Radiology department of KATH for the various radiological support services they offered in patients' management. Dr Ato Quansah deserves special mention for helping with nephrostomy tube placement.

\section{REFERENCES}

1. Iarostki L, Davis A. The Schistosomiasis problem in the world: results of a WHO questionnaire survey. Bull Wld Hlth Org 1981; 59:115-127.

2. Hendrickse RG. Parasitic Diseases. In: Hendrickse RG, Barr DGD and Matthews TS. Eds. Paediatrics in the tropics. Oxford: Blackwell Scientific Publications.1991: pp 695-764

3. Kline MW, Sullivan T. Schistosomiasis. In: Feign RD, Cherry JD Eds. Textbook of Paediatric infectious diseases. Philadelphia: WB Saunders. 3rd edition, 1992:pp 2112-2119.

4. Paperna I. Aquatic weeds, snails and transmission of bilharzias in the new man-made Volta lake in Ghana. Bull de I'I.F.A.N 1969a; A (2) 31:437-499

5. Wright WH. Geographical distribution of schistosomes and their intermediate hosts. In: Ansari N, Ed. Epidemiology and control of schistosomiasis (Bilharziasis). Basel, S. Karger, 1973: pp70-73.

6. Lyons GRL. Schistosomiasis in northern Ghana. Bull Wld Hlth Org 1974; 51(6): 621-632

7. Jordan P, Webbe G. Epidemiology. In: Jordan P, Webbe G, Sturrock RF. Eds. Human schistosomiasis. Wallingford, UK CAB International. 1993: pp87-158

8. Herberg J, Pahari A, Walters S, Levin M. Infectious diseases and the kidney. In: Avner ED, Harmon WE, Niaudet P, Yoshikawa N. Eds. Pediatric nephrology. Berlin Heiddelberg 2009, 6th edition. pp-1273.

9. Sobh MA, Moustafa FE, El-Housseini F et al. Schistosomal specific nephropathy leading to endstage renal failure. Kidney Int 1987; 31:1006-1011.

10. Martinelli R, NoblatAC, Brito E et al. Schistosoma mansoni-induced mesangiocapillary glomerulonephritis; influence of therapy. Kidney Int 1989; 35:1227-1233.

11. Tohon ZB, Mainassara HB, Garba A et al. Controlling Schistosomiasis: Significant Decrease of Anaemia Prevalence One Year after a Single Dose of Praziquantel in Nigerien School children PLoS Negl Trop Dis. 2008 May 28;2(5):e241.

12. McNiece KL, Portman RJ. Hypertension: epidemiology and evaluation. In: Kher KK, Schnaper HW, and Makker SP. Eds. Clinical Pediatric Nephrology. London: Informa Healthcare, 2nd edition, 2006: pp461-480 